\title{
Activation of the HMGB1-TLR4-NF-кB pathway may occur in patients with atopic eczema
}

\author{
YONG WANG ${ }^{1,2^{*}}$, HUI WENG ${ }^{3 *}$, JIAN FEI SONG $^{4}$, YUN HUA DENG $^{5}$, SHUANG LI $^{6}$ and HONG BO LIU ${ }^{1}$ \\ ${ }^{1}$ Department of Laboratory Medicine, The Second Affiliated Hospital of Guilin Medical University, Guilin, \\ Guangxi 541199; ${ }^{2}$ Department of Physiology, Guilin Medical University, Guilin, Guangxi 541004; ${ }^{3}$ Department \\ of Immunology, Tongji Medical College, Huazhong University of Science and Technology, Wuhan, \\ Hubei 430030; ${ }^{4}$ Department of Thoracic and Cardiovascular Surgery, The Second Affiliated Hospital of Guilin \\ Medical University, Guilin, Guangxi 541199; ${ }^{5}$ Department of Dermatology, Tongji Hospital, Tongji Medical \\ College, Huazhong University of Science and Technology, Wuhan, Hubei 430030; ${ }^{6}$ Department of Clinical \\ Laboratory, Nanxishan Hospital of Guangxi Zhuang Autonomous Region, Guilin, Guangxi 541002, P.R. China
}

Received August 8, 2016; Accepted May 12, 2017

DOI: $10.3892 / \mathrm{mmr} .2017 .6942$

\begin{abstract}
High mobility group protein B1 (HMGB1) has been reported to serve important roles in various pathological conditions. Toll-like receptor 4 (TLR4), as one of the HMGB1 receptors, has been reported to be involved in the development of certain inflammatory diseases by activating nuclear factor $\mathrm{NF}-\kappa-\mathrm{B}(\mathrm{NF}-\kappa \mathrm{B})$. However, there are few studies investigating the effects of HMGB1, TLR 4 and $N F-\kappa B$ on human inflammatory dermatoses. In the present study, the distribution and characteristics of HMGB1, TLR4 and NF- $\kappa B$ p65 expression in psoriasis and atopic eczema (AE) were investigated. In addition, immunohistochemical analysis was performed to evaluate their expression and distribution in normal skin, and in patients with AE or psoriasis. Spearman's correlation analysis was used to predicate their relevancy. The present study identified that the p65 level in epithelial nuclei in AE skin was increased compared with normal and psoriasis skin $(\mathrm{P}<0.01)$. The level of extracellular HMGB1 in $\mathrm{AE}$ skin was also increased compared with normal and psoriasis skin $(\mathrm{P}<0.01)$. Meanwhile, TLR4 expression on the epithelial membranes of AE skin was increased compared with psoriasis skin $(\mathrm{P}<0.01)$. Furthermore, the level of extracellular HMGB1 was positively correlated with epithelial membrane TLR4 $(\mathrm{r}=0.3856 ; \mathrm{P}<0.05)$ and epithelial nuclear
\end{abstract}

Correspondence to: Dr Hong Bo Liu, Department of Laboratory Medicine, The Second Affiliated Hospital of Guilin Medical University, 212 Renmin Road, Lingui, Guilin, Guangxi 541199, P.R. China

E-mail: hbliu@glmc.edu.cn

*Contributed equally

Key words: eczema, high mobility group protein B1, inflammation, nuclear factor- $\kappa-\mathrm{B}$, psoriasis, Toll-like receptor 4 p65 $(\mathrm{r}=0.5894 ; \mathrm{P}<0.01)$ in AE skin. These results indicated that the HMGB1-TLR4-NF- $\mathrm{BB}$ signaling pathway is activated in $\mathrm{AE}$ and may account for its pathogenesis, but not in psoriasis. Therefore, HMGB1, TLR4 and NF- $\mathrm{BB}$ p65 have the potential to be targets for the treatment of human inflammatory dermatoses, including AE.

\section{Introduction}

As two of the most common inflammatory dermatoses, atopic eczema (AE) and psoriasis threaten the quality of life and health in humans. AE is an inflammation of the skin characterized by pruritic, papulovesicular and occasional weeping dermatitis (1), and its etiology is hypothesized to be a combination of genetic and environmental factors. It has been identified that AE may sometimes be an allergic reaction to house dust mites (2). Psoriasis is clinically characterized by dry, reddish and silvery-white scaly plaques. Psoriasis is considered to be a primary T-lymphocyte-based immune-pathogenetic disorder $(3,4)$ and runs a chronic course with recurrence caused by environmental factors, including trauma, infection and stress $(5,6)$. Elevated levels of the proinflammatory cytokines interleukin (IL)-17 and IL-22 have been observed in patients with psoriasis $(7,8)$. Psoriasis and AE are hypothesized to be associated with the persistence of local proinflammatory activities $(9,10)$. However, the molecular mechanisms in the development of these diseases remain to be further elucidated.

HMGB1, a 30-kDa nuclear protein referred to one of the most important chromatin proteins, serves as a proinflammatory cytokine mediator, following release by macrophages or monocytes, or during various pathological necrotic conditions $(11,12)$. TLR4, as one of the HMGB1 receptors, is also involved in the induction of inflammatory responses. Extracellular HMGB1 binds to TLR4, which causes myeloid differentiation primary response gene 88 to activate nuclear factor $\kappa \mathrm{B}(\mathrm{NF}-\kappa \mathrm{B})(13)$. Activated $\mathrm{NF}-\kappa \mathrm{B}$ modulates the immune response through the transcriptional regulation of cytokines and chemokines (14). 
It has been recognized that HMGB1 serves significant roles in autoimmunity diseases, epidermal tumors, toxic epidermal necrolysis and Stevens-Johnson syndrome (15-18), and TLR4 and $\mathrm{NF}-\kappa \mathrm{B}$ have been reported to take part in the development of certain tumors and inflammatory diseases $(14,16)$. However, the effects of these mediators on human inflammatory dermatoses have not been elucidated. In the present study, the involvement of the HMGB1-TLR4-NF- $\kappa \mathrm{B}$ signaling pathway in the pathogenesis of $\mathrm{AE}$ and psoriasis was investigated.

\section{Materials and methods}

Tissue specimens. The present study was performed in accordance with The Declaration of Helsinki 1964 and its later amendments, and was approved by the Ethics Board of Tongji Medical College (Wuhan, China). Diagnosis of AE and psoriasis was based on the clinically apparent symptoms and histopathological criteria. The severity of AE and psoriasis were assessed by two well-trained and experienced dermatologists, according to the scoring AD (SCORAD) index and Psoriasis Area and Severity Index (PASI), respectively $(19,20)$. Patients who had an illness duration of $>2$ years and exhibited a moderate-severe condition (SCORAD index, 25-60; PASI score, 6-15) were enrolled. Tissue specimens were obtained from active lesions of patients through cutaneous biopsy following informed consent, including 12 patients with psoriasis vulgaris (5 females and 7 males; age, 22-61 years) and 11 patients with chronic AE (6 females and 5 males; age, 18-54 years). Normal skin specimens were obtained from 10 cases ( 4 females and 6 males; age, 24-57 years) undergoing reconstructive surgery. All specimens were collected between March and December 2010. A therapeutic washout period of 4 weeks for oral medication and 2 weeks for topical treatment was implemented prior to specimen collection. Patients who had a history of other autoimmune diseases, tumors or immunologic deficiency diseases were excluded.

Antibodies and reagents. The antibodies and reagents used in the present study include anti-HMGB1 (cat. no. 2600-1; Epitomics; Abcam, Cambridge, UK), anti-TLR4 (cat. no. ab22048; Abcam), anti-NF-кB p65 (cat. no. SC-7151; Santa Cruz Biotechnology, Inc., Dallas, TX, USA) and REAL ${ }^{\mathrm{TM}}$ EnVision Detection kit (Dako; Agilent Technologies, Inc., Santa Clara, CA, USA).

Immunohistochemistry. The tissue specimen preparation and immunohistochemical procedures were as previously described (16). Tissue specimens were fixed in formalin, embedded in paraffin and sectioned at $4-\mu \mathrm{m}$ thickness. Following deparaffinization, rehydration, antigen retrieval and blocking of non-specific binding, the sections were incubated with primary antibodies (anti-HMGB1, 1:800; anti-TLR4, 1:200; anti-NF- $\mathrm{B}$ p65, 1:200) at $4^{\circ} \mathrm{C}$ overnight. The specimens were subsequently incubated with horseradish peroxidase-conjugated secondary antibodies (1:500; cat. nos. ab6789 and ab6721; Abcam) at room temperature for 45 min. 3,3'-Diaminobenzidine substrate solution was added, and dehydration, hyalinization and mounting were routinely conducted, followed by observation under a Nikon Eclipse Ti-SR microscope and the capturing of images with a Nikon
DS-U3 digital camera (both Nikon Corporation, Tokyo, Japan). Negative controls were obtained by omitting the primary antibodies. A total of two pathologists counted and evaluated the staining independently using a semi-quantitative scoring system as follows (16): 0, No staining; 1, light brown yellow staining; 2, brown staining; and 3, dark brown staining. A total of 10 random fields of view (magnification, $x 400$ ) were counted for each section and average positive expression was scored as follows: $1,<25 \%$; 2 , between 25 and $<50 \%$; 3, between 50 and $<75 \%$; and $4, \geq 75 \%$. The degree of staining and the percentage of expression for each section provided a final score as follows: Negative (-), 0-1 point; weakly positive (+), 2-3 points; moderately positive $(++), 4-6$ points; and strongly positive $(+++)$, $\geq 7$ points.

Statistical analysis. Data are expressed as the mean \pm standard error of the mean. Differences between groups were analyzed using one-way analysis of variance followed by the Bonferroni correction for normally distributed datasets, or by Kruskal-Wallis one-way analysis of variance followed by Nemenyi test for skewed datasets. Spearman's rank correlation coefficient was used for correlation analysis. $\mathrm{P}<0.05$ was considered to indicate a statistically significant difference. Statistical analysis was performed using $\mathrm{R}$ software (version 3.0, GNU Project, Boston, MA, USA).

\section{Results}

Expression of HMGB1 in lesional skin of patients with psoriasis or $A E$. In psoriasis, moderate to strong positive HMGB1 diffuse expression was observed in the nuclei, with weak positive focal expression in the cytoplasm of the squamous epithelium. Extracellular HMGB1 was occasionally present in the intercellular spaces of the epithelium. In the nuclei and cytoplasm of associated inflammatory cells and vascular endothelial cells, moderate positive HMGB1 diffuse expression was observed (Fig. 1A and B).

In $\mathrm{AE}$, there was strong positive HMGB1 diffuse expression in the nuclei, focal weak expression in the cytoplasm and weak positive expression in the intercellular spaces of the squamous epithelium. The nuclei and cytoplasm exhibited diffusive moderate positive HMGB1 expression in the associated inflammatory cells, while exhibiting moderate to strong expression in the vascular endothelial cells (Fig. 1C and D).

In healthy skin, moderate to strong positive HMGB1 expression was observed in the nuclei, with occasional focal expression in the cytoplasm of the squamous epithelium. Little HMGB1 expression was present in the epithelial intercellular spaces. As for inflammatory cells, little HMGB1 expression was observed in the nuclei and cytoplasm, and for vascular endothelial cells, a moderate positive HMGB1 expression was observed (Fig. 1E).

Analysis of variance demonstrated that the HMGB1 expression in epithelial intercellular spaces in AE was significantly increased compared with psoriasis $(\mathrm{P}=0.0023)$ and normal skin $(\mathrm{P}=0.0001)$, and that the HMGB1 expression in epithelial intercellular spaces in psoriasis was markedly increased compared with normal skin (Fig. 1F). There was no statistical difference among the HMGB1 expression in the epithelial nuclei of psoriasis, AE and healthy skin (Fig. 1G). 


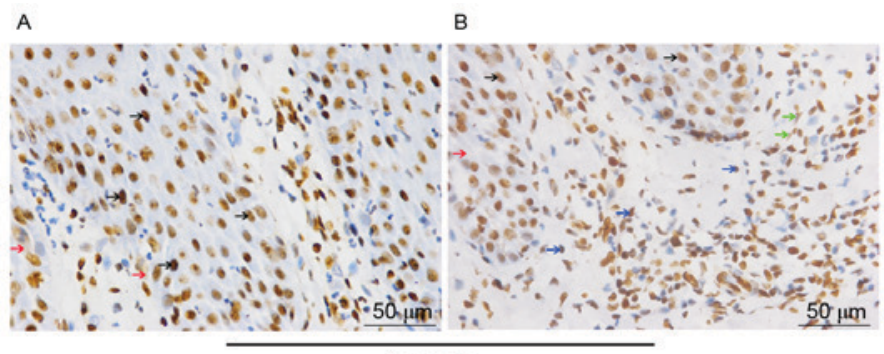

Psoriasis

$\mathrm{E}$

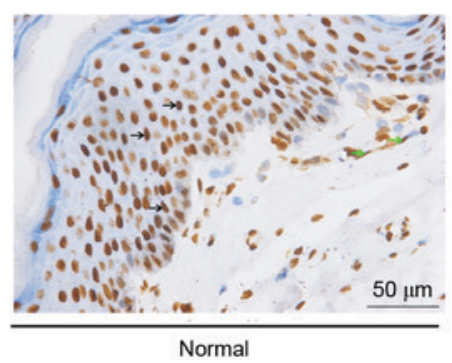

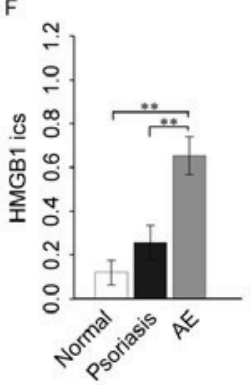

C

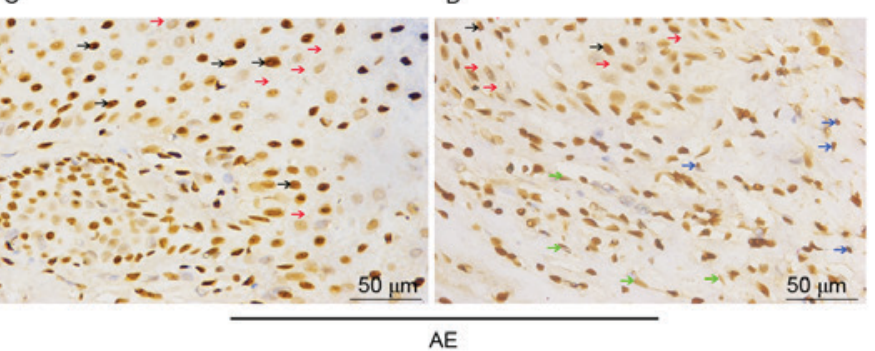

G

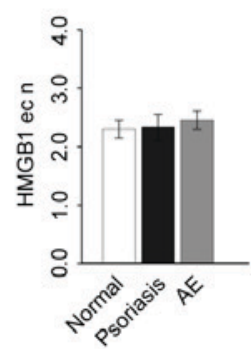

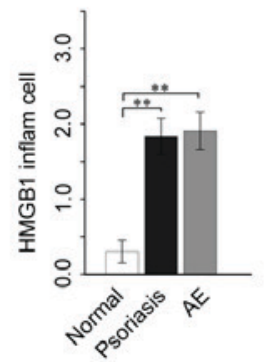

Figure 1. Immunohistochemical analysis of HMGB1 expression in healthy skin and inflammatory dermatoses. HMGB1 expression is located in the nuclei, cytoplasm and epithelial intercellular spaces in (A and B) psoriasis, (C and D) AE and (E) healthy skin. The black arrows represent HMGB1 expression in the ec n; the red arrows represent HMGB1 expression in the ics; the blue arrows represent HMGB1 expression in inflam cells; and the green arrows represent HMGB1 expression in vascular endothelial cells. Magnification, x400. (F) Quantification of HMGB1 in the ics. (G) Quantification of HMGB1 in the ec n. (H) Quantification of HMGB1 in the inflam cells. Data are expressed as the mean \pm standard error of the mean. ${ }^{* *} \mathrm{P}<0.01$. HMGB1, high mobility group protein $\mathrm{B} 1$; ec n, epithelial cell nuclei; ics, intercellular space; inflam, inflammatory; AE, atopic eczema.

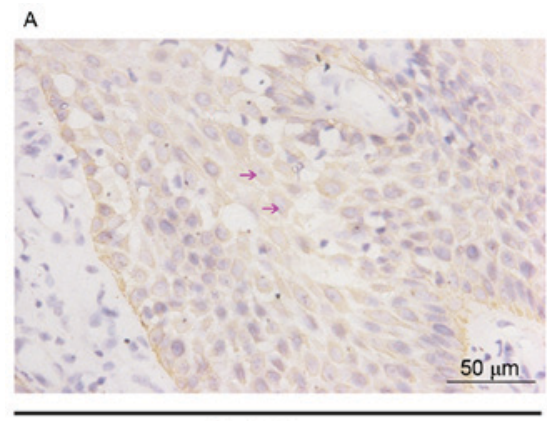

Psoriasis

C

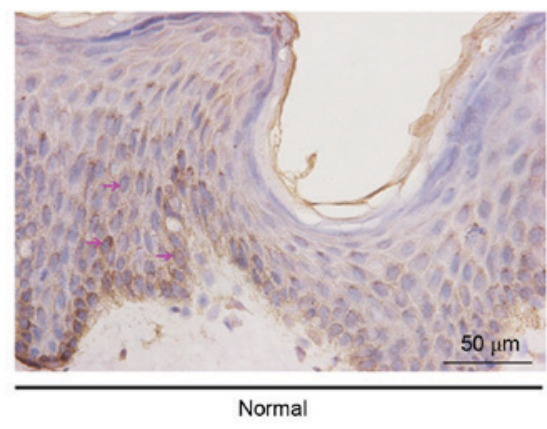

B
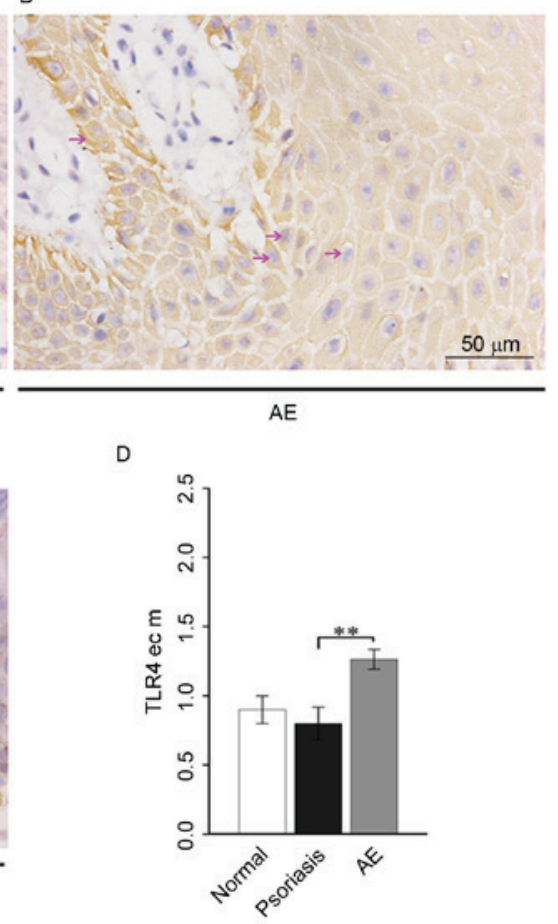

Figure 2. Immunohistochemical analysis of TLR4 expression in (A) psoriasis, (B) AE and (C) healthy skin. The purple arrows represent ec m TLR4 expression. Magnification, $x 400$. (D) Quantification of ec m TLR4 expression. Data are expressed as the mean \pm standard error of the mean. ${ }^{* *} \mathrm{P}<0.01$. TLR4, Toll-like receptor 4; AE, atopic eczema; ec m, epithelial cell membrane.

As for inflammatory cells, HMGB1 expression in psoriasis and $\mathrm{AE}$ was significantly increased compared with healthy skin ( $\mathrm{P}=0.0001$ and $\mathrm{P}=0.0000$, respectively), and the HMGB1 expression in $\mathrm{AE}$ was markedly increased compared with psoriasis (Fig. 1H).
Expression of TLR4 in lesional skin of patients with psoriasis or $A E$. On the squamous epithelium membranes, weak positive TLR4 expression was observed in psoriasis (Fig. 2A), weak to moderate positive expression in AE (Fig. 2B), and weak positive expression in healthy skin (Fig. 2C). Analysis 


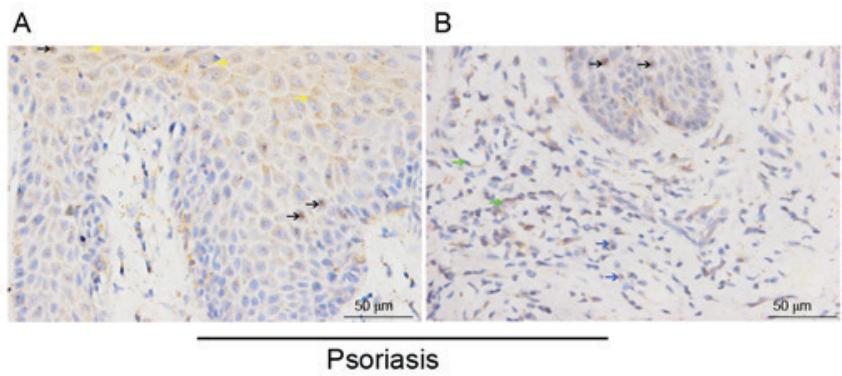

C D

E

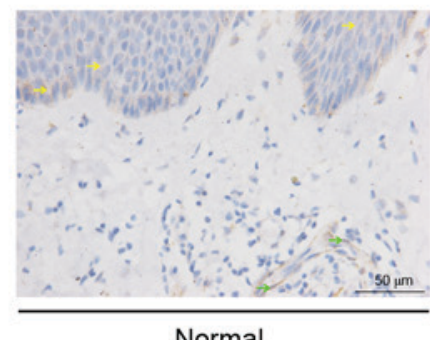

F

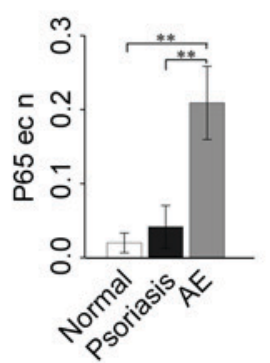

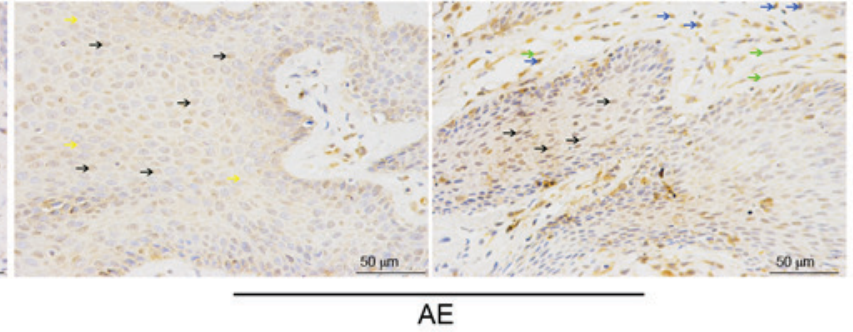

G

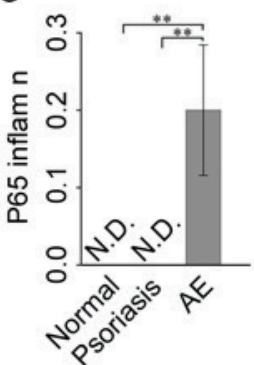

$\mathrm{H}$

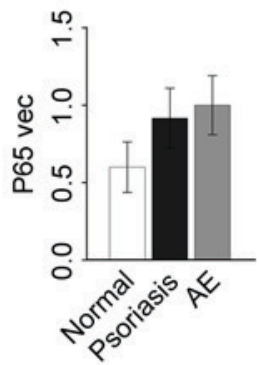

Figure 3. Immunohistochemical analysis of p65 expression in normal skin and inflammatory dermatoses. p65 expression is located in the nuclei and cytoplasm in (A and B) psoriasis, (C and D) AE and (E) healthy skin. The black arrows represent p65 expression in the ec n; the yellow arrows represent p65 expression in the epithelial cytoplasm; the blue arrows represent p65 expression in inflam n; and the green arrows represent p65 expression in vec. Magnification, $\mathrm{x} 400$. (F) Quantification of p65 in the ec n. (G) Quantification of p65 in the inflam n. (H) Quantification of p65 in the vec. Data are expressed as the mean \pm standard error of the mean. ${ }^{* *} \mathrm{P}<0.01$. ec $\mathrm{n}$, epithelial cell nuclei; inflam $\mathrm{n}$, inflammatory cell nuclei; vec, vascular endothelial cells; N.D, not detected; AE, atopic eczema.

of variance demonstrated that epithelial cell membrane TLR4 expression in AE was significantly increased compared with psoriasis $(\mathrm{P}=0.0066)$ and was markedly increased compared with healthy skin, while the TLR4 expression in psoriasis was markedly decreased compared with healthy skin (Fig. 2D).

Expression of $N F-\kappa B$ p65 in lesional skin of patients with psoriasis or $A E$. In psoriasis, sporadic weak positive p65 expression was observed in the nuclei, with weak positive expression in the cytoplasm of the epithelium. Extracellular p65 was occasionally present in the epithelial intercellular spaces. In the associated inflammatory cells, there was focal expression of p65 in the cytoplasm and little expression of p65 in the nuclei. As for vascular endothelial cells, there was a weak positive p65 expression in the cytoplasm; however, no p65 expression in the nuclei (Fig. 3A and B).

In $\mathrm{AE}$ skin, there was a relatively high p65 expression in the nuclei and weak positive expression in the cytoplasm of epithelium. There was also a relatively high p65 expression in the nuclei; however, weak positive focal expression in the cytoplasm of associated inflammatory cells. However, in the vascular endothelial cells, p65 was not observed in the nuclei and exhibited weak positive staining in the cytoplasm (Fig. 3C and D).

In healthy skin, minimal p65 expression was observed in the nuclei, with focal positive expression in the cytoplasm of the epithelium. In the associated inflammatory cells there was little p65 expression in the nuclei. As for vascular endothelial cells, there was sporadic weak positive p65 expression in the cytoplasm (Fig. 3E).

Analysis of variance showed that the p65 expression of epithelial nuclei in AE was significantly increased compared with healthy skin $(\mathrm{P}=0.0066)$ and psoriasis $(\mathrm{P}=0.0082)$, and p65 expression of epithelial nuclei in psoriasis was markedly increased compared with healthy skin (Fig. 3F). The p65 expression of inflammatory cell nuclei in AE was significantly increased compared with healthy skin $(\mathrm{P}=0.0029)$ and psoriasis $(\mathrm{P}=0.0010)$ (Fig. 3G). As for vascular endothelial cells, the p65 level in AE or psoriasis was markedly increased compared with healthy skin (Fig. 3H).

Correlation analysis. In healthy skin and psoriasis tissues, Spearman's rank correlation coefficient analysis demonstrated that there was no significant correlation between the p65 level in epithelial cell nuclei and the HMGB1 level in epithelial intercellular spaces (Fig. 4A) or the epithelial cell membrane TLR4 level (Fig. 4B), or between epithelial cell membrane TLR4 expression and epithelial intercellular space HMGB1 expression (Fig. 4C). In AE tissues, Spearman's rank correlation coefficient analysis demonstrated that the epithelial nuclear p65 expression was positively correlated with the epithelial intercellular space HMGB1 expression ( $r=0.5894$; $\mathrm{P}<0.01$; Fig. 4D). The epithelial nuclear p65 expression was not significantly correlated with the epithelial cell membrane TLR4 expression, although there was a trend $(r=0.1381$; $\mathrm{P}>0.05$; Fig. 4E). However, the epithelial cell membrane TLR4 expression was positively correlated with the epithelial intercellular space HMGB1 expression ( $r=0.3856$; $\mathrm{P}<0.05$; Fig. 4F).

\section{Discussion}

The pathogenesis of $\mathrm{AE}$ and psoriasis remains to be completely elucidated. Despite the clinical distinctions, the two diseases involve the activation of local proinflammatory mediators. The purpose of the present study is to gain an improved understanding of the interactions between HMGB1, TLR4 and NF- $\kappa$ B p65 in the two diseases. The results of the present study support possible roles for these local proinflammatory mediators in the pathogenesis of AE; however, not in psoriasis. 

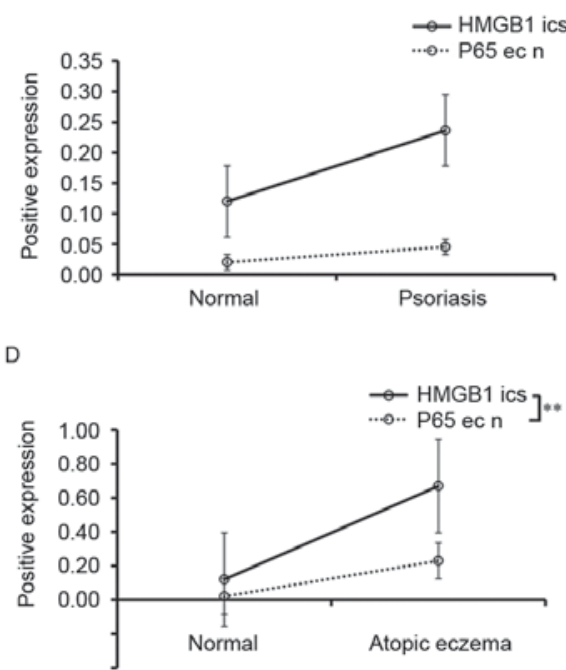

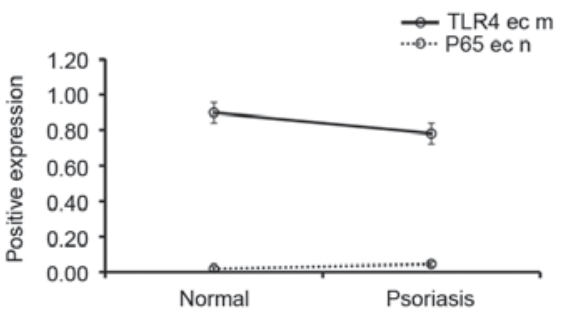

E

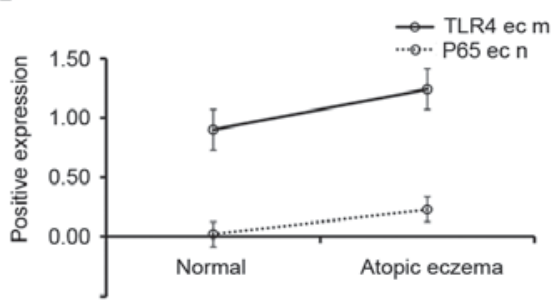

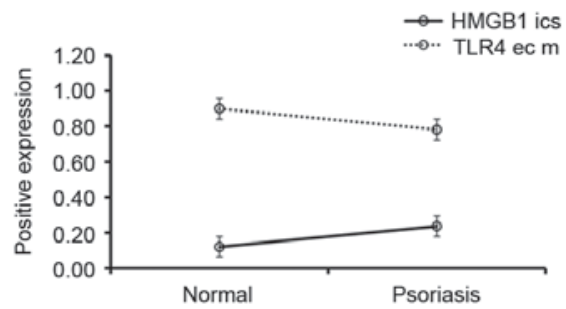

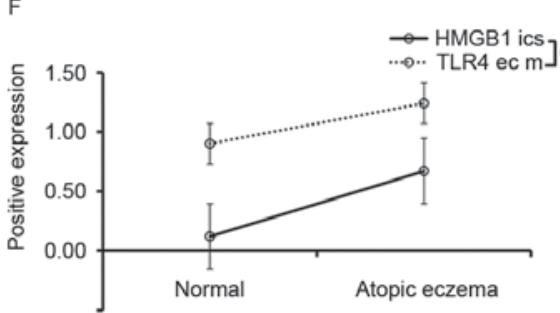

Figure 4. Spearman's rank correlation coefficient analysis of: (A) p65 level in ec n vs. HMGB1 level in ics in normal and psoriasis skin (r=0.1758); (B) p65 level in ec $n$ vs. ec m TLR4 level in normal and psoriasis skin ( $\mathrm{r}=-0.1122)$; (C) ec m TLR4 level vs. HMGB1 level in ics in normal and psoriasis skin ( $\mathrm{r}=-0.3745)$; (D) p65 level in ec n vs. HMGB1 level in ics in normal and eczema skin ( $r=0.5894)$; (E) p65 level in ec $n$ vs. ec m TLR4 level in normal and eczema skin ( $r=0.1381)$; and (F) ec m TLR4 level vs. HMGB1 level in ics in normal and eczema skin ( $\mathrm{r}=0.3856,)^{*} \mathrm{P}<0.05$, ${ }^{* *} \mathrm{P}<0.01$. ec $\mathrm{n}$, epithelial cell nuclei; HMGB1, high mobility group protein B1; ics, intercellular space; ec m, epithelial cell membrane; TLR4, Toll-like receptor 4.

As important inflammatory cytokines and transcription factors, the NF- $\mathrm{B}$ family primarily consists of transcription factor p65, NF- $\kappa \mathrm{B} 1, \mathrm{NF}-\kappa \mathrm{B} 2$, proto-oncogene $\mathrm{c}-\mathrm{Rel}$ and transcription factor RelB $(21,22)$, which are commonly present in the cytoplasm in an inactive state. The activity of $\mathrm{NF}-\kappa \mathrm{B}$ is primarily stimulated by lipopolysaccharide signaling, the $\mathrm{T}$-cell receptor or tumour necrosis factor (23). Activated by various inducers, $\mathrm{NF}-\kappa \mathrm{B}$ protein translocates to the nucleus, regulates the expression of inflammatory factors and becomes a hallmark of inflammatory responses. Increased activation of $N F-\kappa B$ is frequently detected in tumors $(24,25)$, or the location of inflammation in rheumatoid arthritis, inflammatory bowel diseases and asthma, accompanied by the intensified production of proinflammatory factors, including IL-1, IL-6 and TNF (23). The results of the present study demonstrated that the p65 level of epithelial nuclei in AE was increased compared with psoriasis and healthy skin, and the p65 level of epithelial nuclei in psoriasis was markedly increased compared with healthy skin. These results indicated that the $\mathrm{NF}-\kappa \mathrm{B}$ p65-regulated local inflammation was particularly enhanced in AE. By contrast, obscure activation of $\mathrm{NF}-\kappa \mathrm{B}$ signaling was demonstrated in psoriasis. The results were consistent with certain studies demonstrating that enhanced $\mathrm{NF}-\kappa \mathrm{B}$ signaling was observed in atopic dermatitis mouse models (26-28); however, were not consistent with certain other studies demonstrating that NF-kB may also act as a crucial mediator involved in the pathogenesis of psoriasis (29-31). Therefore, continued efforts will be required to identify the roles of $\mathrm{NF}-\kappa \mathrm{B}$ signaling in the two inflammatory skin diseases.

To evaluate the effects of HMGB1 and TLR4 on $\mathrm{NF}-\kappa \mathrm{B}$ p65-regulated inflammation, the significance of the HMGB1-TLR4-NF- $\mathrm{B}$ signaling pathway in the inflammatory dermatoses was investigated. The data demonstrated that there were variant levels of HMGB1 expression in the epithelial intercellular spaces of the tissues, with major expression of extracellular HMGB1 in AE, lesser expression of extracellular HMGB1 in psoriasis and little expression of extracellular HMGB1 in normal skin. These results implied that HMGB1 is released into the extracellular environment in the inflammatory skin diseases, serving as a proinflammatory mediator of the local inflammation. Analysis of variance demonstrated that the expression of extracellular HMGB1 in AE was increased compared with healthy skin, as well as in psoriasis, and extracellular HMGB1 in psoriasis was markedly increased compared with healthy skin, indicating that HMGB1 may be a significant mediator in promoting local inflammation in AE; however, not in psoriasis. These results are inconsistent with the study by Chen et al (17), who observed increased serum HMGB1 levels and altered HMGB1 distribution in the lesional skin of patients with psoriasis vulgaris. It is possible that, in the two studies, different localizations of HMGB1 expression were observed, or patients with different stages and severity assessed by PSAI were enrolled. Therefore, further studies are required to investigate this phenomenon.

TLRs are a type of pattern recognition receptor that recognize structurally conserved molecules derived from microbes and serve a key role in the innate immune system (32). Certain members of the TLR family also recognize HMGB1 and trigger activation of inflammatory responses (33). There is currently sufficient evidence suggesting that HMGB1-TLR4 interactions are important for acute and chronic inflammation-associated pathology (34-36). Panzer et al (37) reported that TLR4 expression was concentrated to the basal layers in healthy skin, whereas it was pronounced in upper layers in atopic dermatitis, contact dermatitis and psoriasis. The present results also revealed that the expression of TLR4 on epithelial cell membranes in AE was increased compared with psoriasis. However, TLR4 on epithelial cell membranes in psoriasis was markedly decreased compared with healthy 
skin. This indicated that TLR4 expression may be a considerable mediator in promoting inflammation in AE; however, not in psoriasis. The results of the present study suggest that extracellular HMGB1 interacts with TLR4 to activate NF- $\mathrm{BB}$ p65 and results in p65-regulated inflammation in AE.

In addition, the present results demonstrated that there was almost the same level of HMGB1 expression in the epithelial nuclei of psoriasis, AE and healthy skin, indicating that the nuclear HMGB1 contributes to the stabilization of DNA and chromosomes, which is consistent with the authors' previous study into the significance of nuclear HMGB1 in certain epidermal tumors (16). The present results also demonstrated that the HMGB1 level in the associated inflammatory cells of $\mathrm{AE}$ and psoriasis was increased compared with healthy skin, and the p65 level in inflammatory nuclei in AE was increased compared with psoriasis, as well as in healthy skin, suggesting that inflammatory skin diseases, particularly AE, may be mediated by inflammatory cells. Furthermore, Spearman's rank correlation coefficient analysis demonstrated that the HMGB1 level in epithelial intercellular spaces was positively correlated with the p65 level in epithelial nuclei, and was also positively correlated with the TLR4 level on epithelial cell membranes in the lesional skin of patients with $\mathrm{AE}$, further indicating that extracellular HMGB1 may bind TLR4 to activate NF-кB p65 and thus promote p65-regulated local inflammation in AE.

In conclusion, the NF- $\kappa \mathrm{B}$ p 65-regulated inflammation was intensified in AE; however, not in psoriasis in the present study. Therefore, the HMGB1-TLR4-NF- $\mathrm{B}$ signaling pathway may be involved in the pathogenesis of $\mathrm{AE}$ and these molecules may be promising targets for attenuating the inflammation in AE. However, there are certain limitations to the present study, as the inflammation intensity is correlated with the stage and severity of skin diseases (17). Only a small number of patients with a certain range of severity were enrolled and only static expressions of three molecules in skin tissue were examined. Therefore, only correlations, not mechanistic information on function, were provided in the present study, and further investigation and validation is required.

\section{Acknowledgements}

The present study was supported by the National Natural Science Foundation of China (grant no. 81460304) and the Guangxi Natural Science Foundation (grant nos. 2015GXNSFAA139197 and 2015GXNSFDA139020).

\section{References}

1. Hanifin JM: Evolving concepts of pathogenesis in atopic dermatitis and other eczemas. J Invest Dermatol 129: 320-322, 2009.

2. Werfel T, Breuer K, Ruéff F, Przybilla B, Worm M, Grewe M, Ruzicka T, Brehler R, Wolf H, Schnitker J and Kapp A: Usefulness of specific immunotherapy in patients with atopic dermatitis and allergic sensitization to house dust mites: A multi-centre, randomized, dose-response study. Allergy 61: 202-205, 2006.

3. Lowes MA, Bowcock AM and Krueger JG: Pathogenesis and therapy of psoriasis. Nature 445: 866-873, 2007.

4. Lew W, Bowcock AM and Krueger JG: Psoriasis vulgaris: Cutaneous lymphoid tissue supports T-cell activation and 'Type 1' inflammatory gene expression. Trends Immunol 25: 295-305, 2004

5. Boyman O, Conrad C, Tonel G, Gilliet M and Nestle FO: The pathogenic role of tissue-resident immune cells in psoriasis. Trends Immunol 28: 51-57, 2007.
6. Griffiths CE and Barker JN: Pathogenesis and clinical features of psoriasis. Lancet 370: 263-271, 2007.

7. Boniface K, Guignouard E, Pedretti N, Garcia M, Delwail A, Bernard FX, Nau F, Guillet G, Dagregorio G, Yssel H, et al: A role for T cell-derived interleukin 22 in psoriatic skin inflammation. Clin Exp Immunol 150: 407-415, 2007.

8. Takahashi H, Tsuji H, Hashimoto Y, Ishida-Yamamoto A and Iizuka H: Serum cytokines and growth factor levels in Japanese patients with psoriasis. Clin Exp Dermatol 35: 645-649, 2010.

9. Kasraie $\mathrm{S}$ and Werfel T: Role of macrophages in the pathogenesis of atopic dermatitis. Mediators Inflamm 2013: 942375, 2013.

10. Valledor AF, Comalada M, Santamaria-Babi LF, Lloberas J and Celada A: Macrophage proinflammatory activation and deactivation: A question of balance. Adv Immunol 108: 1-20, 2010.

11. Dai S, Sodhi C, Cetin S, Richardson W, Branca M, Neal MD, Prindle T, Ma C, Shapiro RA, Li B, et al: Extracellular high mobility group box-1 (HMGB1) inhibits enterocyte migration via activation of Toll-like receptor-4 and increased cell-matrix adhesiveness. J Biol Chem 285: 4995-5002, 2010.

12. Pisetsky D: Cell death in the pathogenesis of immune-mediated diseases: The role of HMGB1 and DAMP-PAMP complexes. Swiss Med Wkly 141: w13256, 2011.

13. Tadie JM, Bae HB, Deshane JS, Bell CP, Lazarowski ER, Chaplin DD, Thannickal VJ, Abraham E and Zmijewski JW: Toll-like receptor 4 engagement inhibits adenosine 5 '-monophosphate-activated protein kinase activation through a high mobility group box 1 protein-dependent mechanism. Mol Med 18: 659-668, 2012.

14. Oh $\mathrm{H}$ and Ghosh S: NF- $\kappa$ B: Roles and regulation in different CD4(+) T-cell subsets. Immunol Rev 252: 41-51, 2013.

15. Harris HE, Andersson U and Pisetsky DS: HMGB1: A multifunctional alarmin driving autoimmune and inflammatory disease. Nat Rev Rheumatol 8: 195-202, 2012.

16. Weng H, Deng Y, Xie Y, Liu H and Gong F: Expression and significance of HMGB1, TLR4 and NF- $\mathrm{KB}$ p65 in human epidermal tumors. Bmc Cancer 13: 311, 2013.

17. Chen T, Guo ZP, Li L, Wang L, Jia RZ, Cao N, Qin S and Li MM: Increased HMGB1 serum levels and altered HMGB1 expression in patients with psoriasis vulgaris. Arch Dermatol Res 305: 263-267, 2013.

18. Nakajima S, Watanabe $H$, Tohyama M, Sugita K, Iijima M, Hashimoto K, Tokura Y, Nishimura Y, Doi H, Tanioka M, et al: High-mobility group box 1 protein (HMGB1) as a novel diagnostic tool for toxic epidermal necrolysis and Stevens-Johnson syndrome. Arch Dermatol 147: 1110-1112, 2011.

19. Oranje AP, Glazenburg EJ, Wolkerstorfer A and de Waard-van der Spek FB: Practical issues on interpretation of scoring atopic dermatitis: The SCORAD index, objective SCORAD and the three-item severity score. Br J Dermatol 157: 645-648, 2007.

20. Langley RG and Ellis CN: Evaluating psoriasis with psoriasis area and severity index, psoriasis global assessment, and lattice system physician's global assessment. J Am Acad Dermatol 51: 563-569, 2004

21. Verma IM, Stevenson JK, Schwarz EM, Van Antwerp D and Miyamoto S: Rel/NF-kappa B/I kappa B family: Intimate tales of association and dissociation. Genes Dev 9: 2723-2735, 1995.

22. Ghosh S, May MJ and Kopp EB: NF-kappa B and Rel proteins: Evolutionarily conserved mediators of immune responses. Annu Rev Immunol 16: 225-260, 1998.

23. Li Q and Verma IM: NF-kappaB regulation in the immune system. Nat Rev Immunol 2: 725-734, 2002.

24. Mayo MW and Baldwin AS: The transcription factor NF-kappaB: Control of oncogenesis and cancer therapy resistance. Biochim Biophys Acta 1470: M55-M62, 2000.

25. Lin A and Karin M: NF-kappaB in cancer: A marked target. Semin Cancer Biol 13: 107-114, 2003.

26. Karuppagounder V, Arumugam S, Thandavarayan RA, Pitchaimani V, Sreedhar R, Afrin R, Harima M, Suzuki H, Nomoto M, Miyashita S, et al: Modulation of HMGB1 translocation and RAGE/NFKB cascade by quercetin treatment mitigates atopic dermatitis in NC/Nga transgenic mice. Exp Dermatol 24: 418-423, 2015.

27. Lee HK, Kim HS, Kim YJ, Kim JS, Park YS, Kang JS, Yuk DY, Hong JT, Kim Y and Han SB: Sophoricoside isolated from Sophora japonica ameliorates contact dermatitis by inhibiting $\mathrm{NF}-\kappa \mathrm{B}$ signaling in B cells. Int Immunopharmacol 15: 467-473, 2013.

28. Kim JH, Kim MH, Yang G, Huh Y, Kim SH and Yang WM: Effects of topical application of Astragalus membranaceus on allergic dermatitis. Immunopharmacol Immunotoxicol 35: 151-156, 2013. 
29. Goldminz AM, Au SC, Kim N, Gottlieb AB and Lizzul PF: NF- $\kappa$ B: An essential transcription factor in psoriasis. J Dermato Sci 69: 89-94, 2013.

30. Yan S, Xu Z, Lou F, Zhang L, Ke F, Bai J, Liu Z, Liu J, Wang H Zhu $\mathrm{H}$, et al: NF- $\kappa \mathrm{B}$-induced microRNA-31 promotes epidermal hyperplasia by repressing protein phosphatase 6 in psoriasis. Nat Commun 6: 7652, 2015

31. Andrés RM, Montesinos MC, Navalón P, Payá $M$ and Terencio MC: NF- $\kappa$ B and STAT3 inhibition as a therapeutic strategy in psoriasis: In vitro and in vivo effects of BTH. J Invest Dermatol 133: 2362-2371, 2013.

32. Kaisho T and Akira S: Toll-like receptor function and signaling. J Allergy Clin Immunol 117: 979-987; quiz 988, 2006.

33. Park JS, Gamboni-Robertson F, He Q, Svetkauskaite D, Kim JY, Strassheim D, Sohn JW, Yamada S, Maruyama I, Banerjee A, et al: High mobility group box 1 protein interacts with multiple Toll-like receptors. Am J Physiol Cell Physiol 290: C917-C924, 2006.

34. Nair AR, Ebenezer PJ, Saini Y and Francis J: Angiotensin II-induced hypertensive renal inflammation is mediated through HMGB1-TLR4 signaling in rat tubulo-epithelial cells. Exp Cell Res 335: 238-247, 2015.
35. Wang FC, Pei JX, Zhu J, Zhou NJ, Liu DS, Xiong HF, Liu XQ, Lin DJ and Xie Y: Overexpression of HMGB1 A-box reduced lipopolysaccharide-induced intestinal inflammation via HMGB1/TLR4 signaling in vitro. World J Gastroenterol 21: 7764-7776, 2015

36. Yang Z, Deng Y, Su D, Tian J, Gao Y, He Z and Wang X: TLR4 as receptor for HMGB1-mediated acute lung injury after liver ischemia/reperfusion injury. Lab Invest 93: 792-800, 2013.

37. Panzer R, Blobel C, Fölster-Holst R and Proksch E: TLR 2 and TLR4 expression in atopic dermatitis, contact dermatitis and psoriasis. Exp Dermatol 23: 364-366, 2014. 